\title{
Determination of L-tryptophan in the presence of ascorbic acid and dopamine using poly (sulfosalicylic acid) modified glassy carbon electrode
}

Xi Ba, Liqiang Luo, Yaping Ding*, Xiao Liu

Department of Chemistry, Shanghai University, Shanghai 200444, P R. China

Corresponding e-mail address: wdingyp@sina.com

\begin{abstract}
:
In this work, a glassy carbon electrode modified with poly-sulfosalicylic acid (PSA/GCE) was prepared by electropolymerization and applied for the determination of L-Tryptophan (L-Trp) in the presence of ascorbic acid and dopamine. The morphologies and interface properties of PSA film were examined by scanning electron microscopy and electrochemical impedance spectroscopy. The electrocatalytic oxidation of L-Trp was investigated on the PSA/GCE using cyclic voltammetry (CV) and differential pulse voltammetry (DPV). The proposed method exhibited wide linear response of $5 \times 10^{-8}$ to $4 \times 10^{-4} \mathrm{M}$ with low detection limit and high selectivity, making it suitable for the analytical purpose.
\end{abstract}

Keywords: Poly-sulfosalicylic acid, L-Tryptophan, Electropolymerization, Differential pulse voltammetry

\begin{abstract}
Introduction
L-Tryptophan (L-Trp) is one of essential amino acids to human and a vital constituent of protein biosynthesis of living organisms [1]. As a biochemical precursor for neurotransmitter serotonin and neurohormone melatonin [2], it is very essential for people with sleep deprivation, anxiety and mood enhancement [3]. Due to the scarce presence of tryptophan in vegetables, LTrp has been commonly added to dietary, food products as food fortifier or pharmaceutical formulations. When improperly metabolized, a waste product will be created in the brain to cause hallucinations and delusions [4]. So, it is necessary to establish a simple, accurate, rapid and inexpensive method for the determination of L-Trp in food, pharmaceutical products and biological fluids.
\end{abstract}

Some methods have been developed for the determination of L-Trp, such as spectroscopy [5], high-performance liquid chromatography [6], fluorometric methods [7], capillary electrophoresis [8] and electroanalysis [9]. Among them, electrochemical techniques have gained much more attention for its high sensitivity, high accuracy, simple operation mode and low cost. However, the voltammetric response of L-Trp at bare electrode is not optimal because of sluggish electron transfer processes and high overpotential [10]. Hence, many efforts have been devoted to promoting the electron transfer and reducing the overpotential for the electrochemical oxidation of L-Trp [11-16].
Recently, various modified electrodes have been reported for the determination of L-Trp, such as poly 4-aminobenzoic acid [11], poly glutamic acid [12], 1-[4-(ferrocenyl ethynyl) phenyl]-1-ethanone [13], Ni (II)/ACDA-AuNP$\mathrm{Au}$ [14], gold nanoparticles [15] and nano$\mathrm{TiO}_{2} /$ ferrocence carboxylic acid [16]. Among these electrodes, polymer film modified electrodes show unique properties and an enhanced response for the application in samples [17]. Since the thickness, permeation and charge transport characteristics of the polymeric films can be adjusted by the potential and current applied, the fabrication of conducting polymer film is flexible and controlled [18].

In this work, a novel sensor of L-Trp based on poly (sulfosalicylic acid) film (PSA) modified glassy carbon electrode by electrochemical polymerization (PAS/GCE) was developed. Because of high electron density of carbonyl and sulfonic groups in sulfosalicylic acid molecule $\left(\mathrm{COO}^{-}\right.$and $\left.\mathrm{SO}_{3}{ }^{2-}\right)$, the PSA film has high concentrations of negatively-charged surface-functional groups. The modified electrode showed excellent electrocatalytic properties with obvious reduction of overpotential and enhancement of oxidation current and was applied for the detection of LTrp coexisting with some possible interfering substances. 


\section{Experiment}

Electrochemical measurements were carried out on a $\mathrm{CHI} 660 \mathrm{C}$ electrochemical workstation (Shanghai Chenhua Co., Ltd., China) with a conventional three-electrode system. A clean GCE was immersed in $0.1 \mathrm{M}$ PBS ( $\mathrm{pH} 5.5)$ containing $10 \mathrm{mM}$ sulfosalicylic acid using cyclic voltammetry from -1.0 to $2.0 \mathrm{~V}$ at $100 \mathrm{mv} \mathrm{s}^{-1}$ for 30 cycles and washed with double distilled water for use.

\section{Characterization}

To investigate the morphology of the modified electrode, we performed scanning electron microscope (SEM). Fig. 1 shows the typical morphology of PSA film, indicating that the film has a fine cluster-like structure. SEM image of PSA film was very uniform, which verified that the PSA film was successfully polymerized on the electrode surface and the structure of PSA film could improve the effective electrode surface.

Electrochemical impedance spectroscopy (EIS) was used as a powerful technique to study the interface properties of the electrode surfaces. Fig. 2 shows the typical Nyquist diagrams of the EIS in $5.0 \mathrm{mM}\left[\mathrm{Fe}(\mathrm{CN})_{6}\right]^{3-14-}$ solution at the bare GCE (a), PSA/GCE (b). Compared with the bare GCE (curve a), the electron-transfer resistance $\left(R_{e t}\right)$ for PSA/GCE was larger (curve b).This may be ascribed to the electrostatic repulsion force between the negatively charged $\left[\mathrm{Fe}(\mathrm{CN})_{6}\right]^{3-14-}$ and poly (sulfosalicylic acid) film [19]. The change of the $R_{\text {et }}$ value suggests that the PSA film is assembled onto the surface of GCE.

\section{Electrochemical behavior of L-Trp at PSA/GCE}

The electrocatalytic activity of PSA/GCE was demonstrated by a comparison of the differential pulse voltammograms (DPVs) of different electrodes in 0.1 M PBS (pH 3.5). Fig. 3 shows DPVs of $1 \times 10^{-4} \mathrm{M}$ L-Trp at the bare GCE (b), PSA/GCE(c). It can be seen that the oxidation peak current of L-Trp at PSA/GCE is enhanced and the peak is sharper than the bare GCE (curve b). The increase of peak current maybe attributed to the electrostatic interaction between the negatively charged PSA and the positively charged L-Trp in $0.1 \mathrm{M}$ PBS (pH 3.5) [12]. Meanwhile, the peak potential shifted negatively from $0.91 \mathrm{~V}$ to $0.79 \mathrm{~V}$, indicating that the PSA film has good electrocatalytic activity towards L-Trp.

\section{Calibration curve and interferences}

Under the optimum conditions, the electrochemical behaviors of different concentrations of L-Trp were studied. From Fig. $4(A)$, the change of DPVs indicates that the oxidative peak current $\left(I_{p}\right)$ has linear relationship with the concentration (c) of L-Trp. In the range from $5.0 \times 10^{-8}$ to $4.0 \times 10^{-4} \mathrm{M}$, two linear regression equations $I_{p}(\mu \mathrm{A})=0.2524+$ $0.3922 c(\mu \mathrm{m}), I_{p}(\mu \mathrm{A})=4.7947+0.0369 c(\mu \mathrm{m})$ were obtained with the correlation coefficients of 0.9963 and 0.9943 consequently.

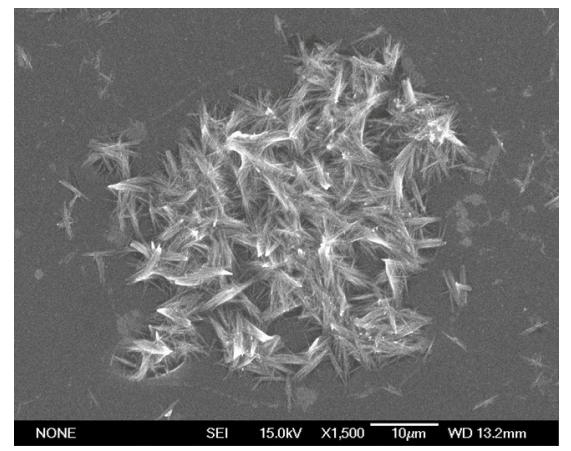

Fig. 1. Scanning electron microscope of the PSA film.

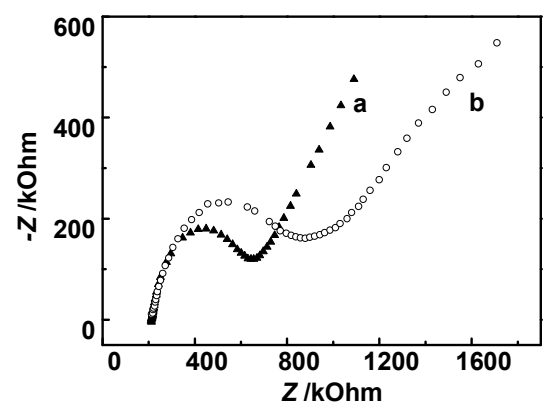

Fig. 2. Electrochemical impedance spectra of bare GCE (a), PSA/GCE (b).

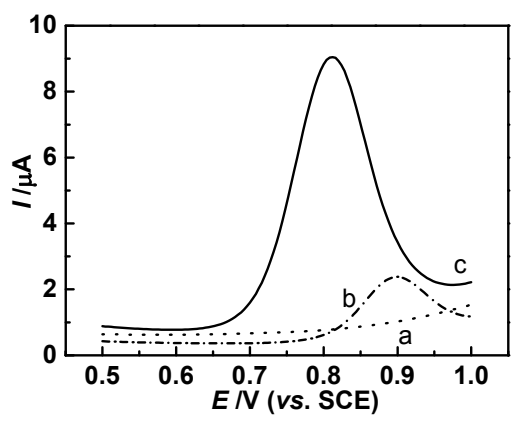

Fig. 3. DPVs of $1 \times 10^{-4} \mathrm{M} L-T r p$ in $0.1 \mathrm{M} P B S(\mathrm{pH}$ 3.5) at the bare GCE (b), PSA/GCE(c) and the bare GCE in the absence of L-Trp (a). 

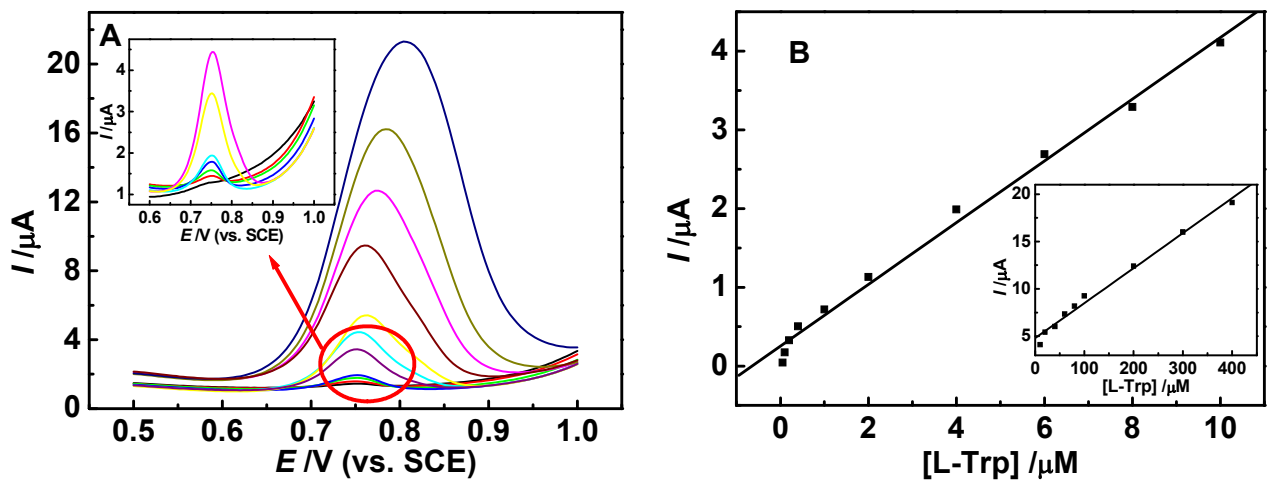

Fig. 4. (A) DPVs of the PSA/GCE at different L-Trp concentrations from $5 \times 10^{-8} \mathrm{M}$ to $4 \times 10^{-4} \mathrm{M}$. The above inset is the amplifying DPV of L-Trp from $5 \times 10^{-8} \mathrm{M}$ to $1 \times 10^{-5} \mathrm{M}$. (B) the calibration curve for the determination of L-Trp. Insert: the plot of the peak current vs. L-Trp concentration

The detection limit $(\mathrm{S} / \mathrm{N}=3)$ and the sensitivity was calculated to be $6.8 \times 10^{-9} \mathrm{M}$ and 1913.63 $\mu \mathrm{A} \cdot \mathrm{mM}^{-1} \cdot \mathrm{cm}^{-2}$ respectively.

To evaluate the influences of some potential interference on the determination of L-Trp, various foreign species were added into $0.1 \mathrm{M}$ PBS containing L-Trp $\left(1 \times 10^{-6} \mathrm{M}\right)$, such as cysteine, alanine, phenylalanine, glutamic. The results indicate the interference effects of the above analytes toward response of L-Trp are negligible when the concentration is more than 100-times. Furthermore, there are some important biological substances like AA and DA often coexisting with L-Trp in biological samples. Fig.5 describes the DPVs of different concentrations of L-Trp at PSA/GCE in the presence of $A A$ and DA. It can be seen that three well-separated peaks presented at the detached potentials, indicating that $A A$ and DA had no interference for the detection of L-Trp. In addition, the peak currents of three compounds increased synchronously with the increasing of concentrations of L-Trp, AA and DA, implying that PSA/GCE can be also applied for the simultaneous determination of L-Trp, AA and DA.

Table 1 displays analytical results of the proposed method and were compared with other electrochemical methods reported previously for detecting L-Trp. It can be seen that the electrochemical performance of PSA/GCE here is favorable and the electrochemical sensor would be very suitable for practical L-Trp detection.

\section{Conclusions}

A novel sensor of L-Trp was prepared with polysulfosalicylic acid modified glassy carbon electrode by electropolymerization in this work. The modified electrode showed wide linear concentration range, low detection limit and high selectivity. In addition, the method can be applied for the measurement of L-Trp in the presence of $A A$ and DA. The results showed that PSA/GCE had good analytical performance, which can be used for routine analysis of L-Trp in biological samples.

Tab. 1: Comparisons of the proposed PSA/GCE performance with those previously reported

\begin{tabular}{|c|c|c|}
\hline Method & $\begin{array}{c}\text { Dynamic } \\
\text { ranges }(\mu \mathrm{M})\end{array}$ & $\begin{array}{c}\text { Detection } \\
\text { limits }(\mu \mathrm{M})\end{array}$ \\
\hline 4-ABA/GCE[11] & $1-100$ & 0.2 \\
\hline PGA/CNTPE[12] & $0.05-100$ & 0.01 \\
\hline 4-FEPE/CPE[13] & $0.85-63.4$ & 0.56 \\
\hline $\begin{array}{c}\text { AuNP- } \\
\text { CNT/GCE[14] }\end{array}$ & $0.03-2.5$ & 0.01 \\
\hline Au-NPs/GCE[15] & $0.09-500$ & 0.08 \\
\hline Present work & $0.05-400$ & 0.0068 \\
\hline
\end{tabular}

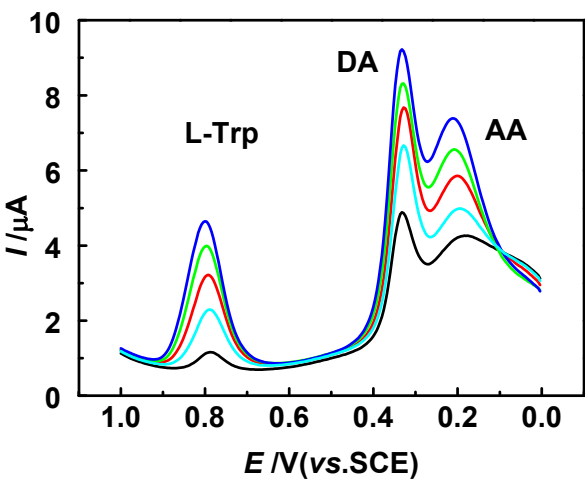

Fig. 5. Differential pulse voltammograms of L-Trp $(1-20 \mu M)$ in $0.1 \mathrm{M}$ PBS $(\mathrm{pH} 3.5)$ in the presence of ascorbic acid (20-200 $\mu \mathrm{M})$ and dopamine (1-20 $\mu \mathrm{M})$. 


\section{Acknowledgements}

This work was supported by the National Natural Science Foundation of China (No. 20975066, 41140031, 61171033), Leading Academic Discipline Project of Shanghai Municipal Education Commission (J50102).

\section{References}

[1] O. Takikawa, Biochemical and medical aspects of the indoleamine 2,3-dioxygenase-initiated Ltryptophan metabolism, Biochemical and Biophysical Research Communications 338, 1219 (2005); doi:10.1016/j.bbrc.2005.09.032

[2] A. Carlsson, M. Lindqvist, Dependence of 5-HT and catecholamine synthesis on concentrations of precursor amino-acids in rat brain, N.S.Arch.Pharmacol 303, 157-164 (1978); doi: 10.1007/BF00508062

[3] P. Prabhu, R. Suresh Babu, S. Sriman Narayanan, Electrocatalytic oxidation of Itryptophan using copper hexacyanoferrate film modified gold nanoparticle graphite-wax electrode, Colloids and Surfaces B: Biointerfaces 87, 103-108, doi:10.1016/j.colsurfb.2011.05.008

(2011);

[4] W. Kochen, H. Steinhart, L-tryptophan current prospects in medicine and drug safety, deGruyter, Berlin, 1994.

[5] D.M. Reynolds, Rapid and direct determination of tryptophan in water using synchronous fluorescence spectroscopy, Water Research 37, 3055-3060, (2003); doi:10.1016/S00431354(03)00153-2

[6] H.P. Fitznar, J.M. Lobbes, G. Kattner, Determination of enantiomeric amino acids with high-performance liquid chromatography and precolumn derivatisation with o-phthaldialdehyde and $\mathrm{N}$-isobutyrylcysteine in seawater and fossil samples (mollusks), Journal of Chromatography A 832, 123-132, (1999); doi:10.1016/S00219673(98)01000-0

[7] E. Sikorska, A. Gliszczynskaswiglo, M. Insinskarak, I. Khmelinskii, D. Dekeukeleire, M. Sikorski, Simultaneous analysis of riboflavin and aromatic amino acids in beer using fluorescence and multivariate calibration methods, Anal. Chim. Acta 613, 207-217, (2008); doi: 10.1016/j.aca.2008.02.063

[8] T. Bo, J. Pawliszyn, Characterization of bovine serum albumin-tryptophan interaction by capillary isoelectric focusing with whole column imaging detection, J. Chromatogra. A 1105, 25-32, (2006); doi:10.1016/j.chroma.2005.08.092

[9] X.F. Tang, Y. Liu, H.Q Hou, T.Y You, Electrochemical determination of L-Tryptophan, L-Tyrosine and L-Cysteine using electrospun carbon nanofibers modified electrode, Talanta 80 , 2182-2186, doi:10.1016/j.talanta.2009.11.027
[10] S.M. MacDonald, S.G. Roscoe, Electrochemical oxidation reactions of tyrosine, tryptophan and related dipeptides, Electrochim. Acta 42, 11891200, (1997); doi:10.1016/S00134686(96)00285$\mathrm{X}$

[11] K.J. Huang, C.X. Xu, W.Z. Xie, W.Wang, Electrochemical behavior and voltammetric determination of tryptophan based on 4aminobenzoic acid polymer film modified glassy carbon electrode, Colloid Surface B 74,167-171, (2009); doi:10.1016/j.colsurfb.2009.07.013

[12] X. Liu, L.Q. Luo, Y.P. Ding, D.X. Ye, Polyglutamic acid modified carbon nanotube-doped carbon paste electrode for sensitive detection of L-tryptophan, Bioelectrochemistry 82, 38-45, (2011); doi:10.1016/j.bioelechem.2011.05.001

[13] J. B. Raoof, R. Ojani, H. Karimi-Maleh, Carbon paste electrode incorporating 1-[4-(ferrocenyl ethynyl) phenyl]-1-ethanone for electrocatalytic and voltammetric determination of tryptophan, Electroanalysis 20, 1259-1262, (2008); doi:10.1002/elan.200704176

[14] M.B. Gholivand, A. Pashabadi, A. Azadbakht, S. Menati, A nano-structured Ni(II)-ACDA modified gold nanoparticle self-assembled electrode for electrocatalytic oxidation and determination of tryptophan, Electrochimica Acta 56, 4022-4030, (2011); doi:10.1016/j.electacta.2011.02.009

[15] C.Y Li, Y. Ya, G.Q. Zhan, Electrochemical investigation of tryptophan at gold nanoparticles modifiedelectrode in the presence of sodium dodecylbenzene sulfonate, Colloids and Surfaces B: Biointerfaces 76, 340-345, (2010); doi:10.1016/j.colsurfb.2009.11.017

[16] J.-B. Raoof, R. Ojani,M. Baghayeri, Simultaneous electrochemical determination of glutathione and tryptophan on a nano- $\mathrm{TiO}_{2} /$ ferrocene carboxylic acid modified carbon paste electrode, Sens. Actuators B 143, 261-269, (2009); doi:10.1016/j.snb.2009.08.046

[17] G.N. Chen, Z.F. Zhao, X.L.Wang, J.P. Duan,H.Q. Chen, Electrochemical behavior of tryptophan and its derivatives at a glassy carbon electrode modified with hemin, Anal. Chim. Acta 452, 245254, (2002); doi:10.1016/s0003-2670(01)01470-2

[18] X.H. Lin, Y.F. Zhang, W. Chen, P. Wu, Electrocatalytic oxidation and determination of dopamine in the presence of ascorbic acid and uric acid at a poly ( $p$-nitrobenzenazo resorcinol) modified glassy carbon electrode, Sens. Actuators. B 122, 309-314, (2007); doi:10.1016/j.snb.2006.06.004

[19] J.P Li, J.G Yu, Q.Y. Lin Amperometric nonenzymatic determination of glucose free of interference based on poly (sulfosalicylic acid) modified nickel microelectrode, Analytical Letters 43, 631-643, doi:10.1080/00032710903406896
(2010); 